\title{
Poikilodermatous mycosis fungoides: clinical and histopathological analysis of a case and literature review
}

\author{
Oleg Pankratov ${ }^{1}$, Svetlana Gradova ${ }^{1}$, Svetlana Tarasevich ${ }^{2}$, Valentin Pankratov³
}

\begin{abstract}
Poikilodermatous mycosis fungoides is a rare distinct clinical variant of cutaneous T-cell lymphoma (CTCL), formerly referred to as poikiloderma vasculare atrophicans or parapsoriasis variegata. Mycosis fungoides (MF) is a malignant neoplasm of T-lymphocyte origin, most commonly memory $\mathrm{CD}_{4}+\mathrm{T}$-cells. We report here a patient with generalized poikilodermatous skin lesions whose diagnosis of mycosis fungoides was made only a few years after the onset of his disease due to its bizarre clinical behavior and a natural reluctance to diagnose this disease in children and adolescents. The variability of atypical clinical presentations of MF and its similarity to benign inflammatory and noninflammatory skin disorders may become a source of considerable confusion and controversy, challenging a dermatologist to make a precise diagnosis. Therefore, scrupulous clinicopathological correlation is an absolute necessity.
\end{abstract}

Keywords: mycosis fungoides, poikilodermatous variant, case report

Received: 27 March 2015 | Returned for modification: 5 April 2015 | Accepted: 12 May 2015

\section{Background}

Poikilodermatous mycosis fungoides is a rare distinct clinical variant of cutaneous T-cell lymphoma (CTCL), formerly referred to as poikiloderma vasculare atrophicans or parapsoriasis variegata. Mycosis fungoides (MF) is a malignant neoplasm of T-lymphocyte origin, most commonly memory $\mathrm{CD}_{4}+\mathrm{T}$-cells (1-3). Its classic (Alibert) variant presents a chronic, slowly progressing disease with erythematous scaly patches at the onset that gradually evolve over time into infiltrated plaques and tumors. Apart from this classic form, there are poikilodermatous and erythrodermic variants (the latter should not be confused with Sézary's syndrome). There are also a wide range of rare atypical presentations including hypo- and hyperpigmented, verrucous, hyperkeratotic, follicular, lichenoid papular, palmoplantar psoriasiform, granulomatous, vesicular, bullous, and pustular variants, which have been described in the literature (1). These are clinically unusual cases that run a similar course to that of classic MF.

In 1906 the pioneering American pediatrician Abraham Jacobi described a complex dermatologic disease characterized by telangiectasia, pigmentation, and atrophy, which he subsequently termed poikiloderma vasculare atrophicans (PVA).

Formerly, poikiloderma vasculare atrophicans of Jacobi was considered a separate clinical entity or a premalignant condition. Later, it was believed that PVA represented a stage or an outcome of various dermatoses, such as mycosis fungoides, parapsoriasis, dermatomyositis, scleroderma, lupus erythematosus, lichen ruber planus, genodermatoses, and so on. However, in recent years these opinions have been challenged by many competent dermatologists. Nowadays poikiloderma vasculare atrophicans is recognized as a clinical variant of patch stage MF (4-6); and poikilodermatous findings on non-sun-exposed areas should be considered MF until proven otherwise.

Poikilodermatous MF is usually characterized by the development of large plaques or generalized skin involvement (7-9).
However, at the onset of the disease the lesions may present either small plaques or papules arranged in a net-like pattern, which makes it very similar to lichen ruber planus. The typical patches often show a predilection to the major flexural areas and trunk, and present with erythema, mild scaling, mottled dyspigmentation (hyper- and hypopigmentation) with atrophy, and telangiectases. As a result of atrophy and thinning, the patient's skin surface may be reminiscent of "cigarette paper." As opposed to classic MF, poikilodermatous lesions are generally asymptomatic or mildly pruritic and are usually stable or slowly increasing in size $(10,11)$. Lymph node enlargement is a frequent finding in patients with MF, but it does not necessarily correlate with histological lymphomatous involvement. Dermatopathic lymphadenitis is often observed in such patients (12).

The first manifestation of poikilodermatous MF usually occurs at an earlier age than that of classic MF, and a male predominance was reported for both forms.

Histopathology of poikilodermatous lesions discloses an atypical T-cell infiltrate in the papillary dermis, often with evident epidermotropism $(1,13)$. However, Pautrier microabscesses are not as common in comparison to classic MF. Melanophages and melanin incontinence are also observed, along with ectasia of the superficial dermal vessels and epidermal atrophy.

Immunohistological staining commonly shows either a prevalence of the $\mathrm{CD}_{4+}+\mathrm{CD} 8-$ pattern or CD8+, CD4- immunophenotype, which is more often seen in hypopigmented variants of MF.

\section{Case report}

Mycosis fungoides is the most common form of cutaneous T-cell lymphoma. We present here a patient with its uncommon poikilodermatous variant.

A 29-year-old Caucasian man presented to the City Clinical Skin and Venereal Diseases Clinic in November 2014 with an 18year history of a diffuse skin affection involving his trunk, neck, 
and extremities. The first eruption appeared on his skin at age eleven. At that time there were a few separate well-defined asymptomatic hypopigmented patches on his chest and left shoulder, which resolved spontaneously without treatment. The patient could not recall any cause that could have triggered the onset of his disease with the exception of sun exposure, which had occurred immediately before the appearance of the first manifestation. However, he denied any possibility of sunburn because his skin had always easily tanned prior to the disease.

After a while, a similar eruption reappeared. However, this time the lesions were persistent and the circumstances forced him to seek medical help. Based on the clinical findings (asymptomatic well-defined hypopigmented patches without obvious atrophy), a diagnosis of vitiligo was made.

At age fourteen, these patches transformed into hyperpigmented plaques with slight atrophy. Thus, the diagnosis was changed to morphea. After that, the patient underwent several courses of treatment for morphea, but they appeared to have only a temporary benefit. The lesions disappeared at one site and simultaneously arose at another. Finally, there was a gradual spread of the skin problems in recent years, although they remained almost asymptomatic throughout the progression. The patient mentioned only a slight burning sensation appearing in wintertime within the last 2 years, which was alleviated by emollients and short courses of medium-strength topical corticosteroids.

Later, in 2012, the skin lesions suddenly changed their appearance and began to spread relatively quickly. The patient's clinical examination at that time showed widespread hyperpigmented patches and small papules with a tendency towards a net-like arrangement within the same distribution area. These papules were not typical planar ones, but had a distribution and arrangement highly suggestive of a diagnosis of lichen ruber planus. Considering those changes, a punch biopsy was performed, and according to the histopathological findings a diagnosis of lichen ruber planus was confirmed (although immunohistological studies were not performed at that time). Although the patient has received a few courses of treatment for lichen ruber planus in the last 3 years, they have shown no efficacy.

Currently, the patient's general health is unimpaired. Although he is rather thin, he denies any episodes of weight loss. The pa-

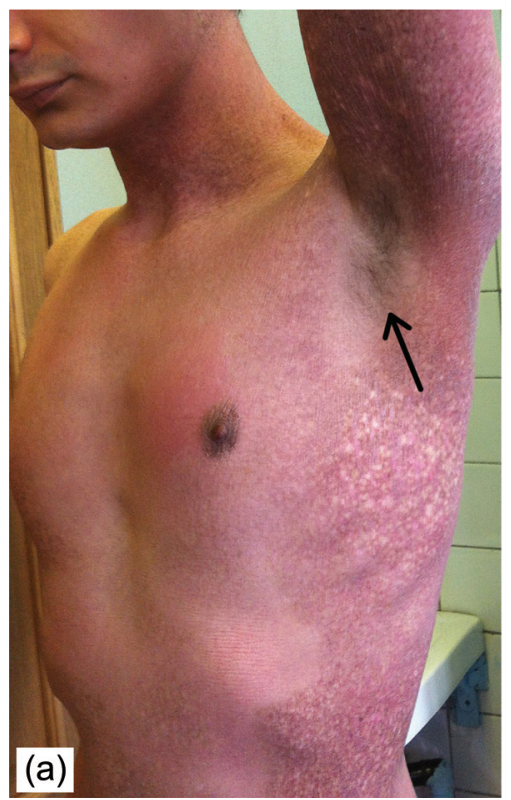

tient complains of no symptoms apart from dry skin, which worsens in winter, and soreness and irritation of the skin after sun exposure in summer.

Data collected from patient's mother revealed some interesting facts that may contribute to the diagnosis and may help in defining the causes of his disease. She told us that she delivered her only son at term, but during the pregnancy she experienced oligohydramnios and the boy was born with neonatal hypotrophy, weighing barely $2.3 \mathrm{~kg}$. Apart from this, at birth her son was covered with what she called a "coat," which began to exfoliate within the first days, and in a week his skin became "normal" and remained healthy until age eleven. Unfortunately, the exact diagnosis is unknown because the documents were lost. However, taking everything into account, the patient might have suffered from one of the ichthyosiform dermatoses. In addition, he had congenital angiomata cavernosum (presumably, according to mother's description) localized on the left earlobe, which was treated with radiotherapy at the age of 3 months. With the exception of these details, the patient's past medical and family histories were non-contributory. The patient's history also did not reveal any occupational hazards.

Physical examination revealed bilateral axillary lymphadenopathy. A few separate slightly enlarged lymph nodes showing no tenderness to palpation were found in both axillae. The entire surface of the skin was very thin, crinkled, and scaly, and had a characteristic wrinkled, “cigarette-paper” appearance. The skin affections had a diffuse distribution with only several small islands of uninvolved skin on the trunk and lower extremities (Fig. 1).

The palms, soles, scalp, and face (excluding a single plaque over the left eyebrow) seemed to be spared. The hair on the head, in the axilla, and in the groin was also preserved, although it appeared thin. At the same time, lanugo hair (equally on healthy skin and on the affected areas) was absent over the entire body surface. The fingernails and toenails were intact. The skin lesions presented confluent poikilodermatous patches and plaques with mottled hyper- and hypopigmentation, atrophy, and telangiectasias. Almost all of these patches, especially those located on the thighs and the lateral aspects of the trunk, were also remarkable for the net-like distributed plane lichenoid papules. A few ill-defined erythematous patches could be observed on the ante-

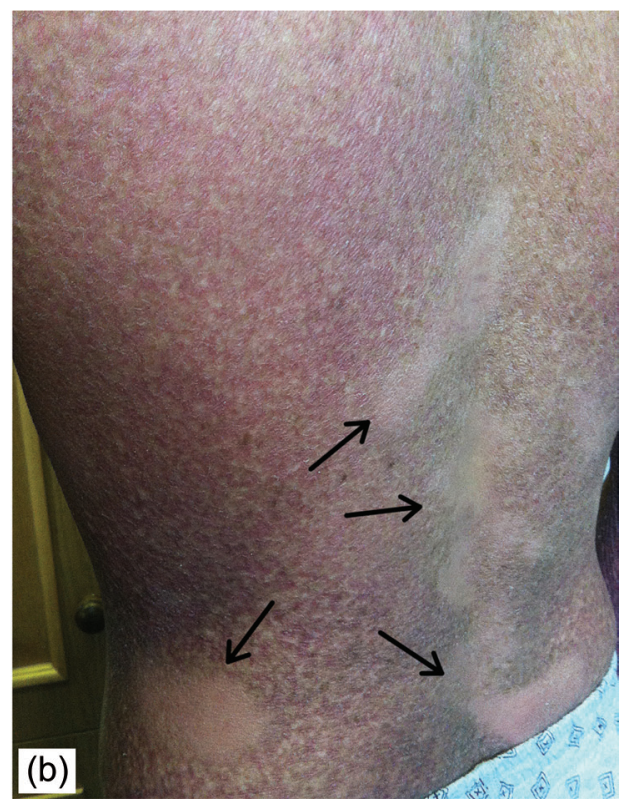

Figure 1 | Diffuse poikilodermatous skin lesions. (a) Spared axillary hair; (b) small islands of uninvolved skin. 
rior chest and in the paraumbilical area. They were both visibly and palpably slightly infiltrated. The anterior aspects of the shins showed several confluent plaques with a grayish-brown tint and evident infiltration.

It should be noted that the earlobe that had been exposed to Xrays in infancy had shrunk and almost disappeared, whereas the other one stayed absolutely intact (Fig. 2).

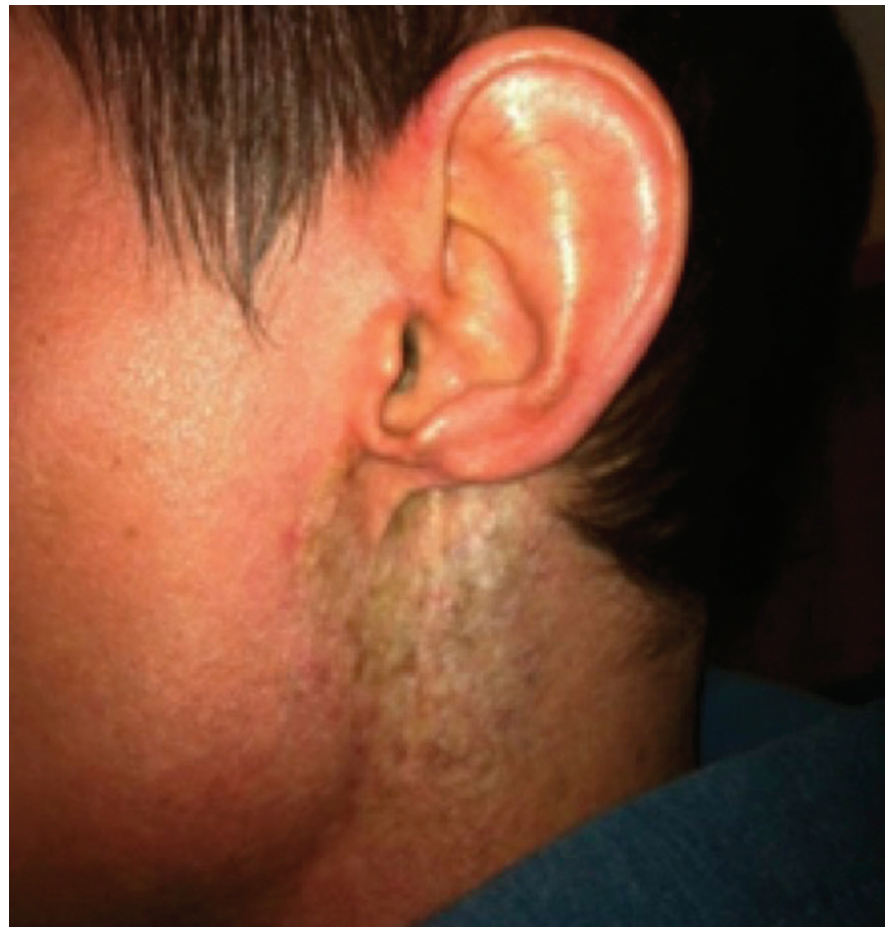

Figure 2 | Shrunk earlobe and the scar that remained after exposure to X-rays.

Taking into consideration the aforementioned features and the past medical history, we were inclined to regard the condition as a rare poikilodermatous form of MF. Because the patient had such diversified skin lesions, it was decided to obtain four punch biopsy specimens from representative areas: an erythematous patch on the anterior aspect of the chest, a typical poikilodermatous patch on the right flank, a lichenoid papule on the right thigh, and the plaque on the anterior aspect of the left shin (Fig. 3).

Histologically, the first erythematous patch showed thickened epidermis with marked acanthosis, adopting a psoriasiform appearance, and focal parakeratosis. There was a superficial lymphohistiocytic infiltrate surrounding small blood vessels with an obvious tendency to palisade along the basal membrane of the epidermis. Pigmentary incontinence and basal cell hydropic degeneration were noted in some fields. Within the epidermis, there were a relatively small number of atypical lymphoid cells with a clear halo, distributed singly and in clusters. However, clear Pautrier microabscesses were absent (Fig. 4).
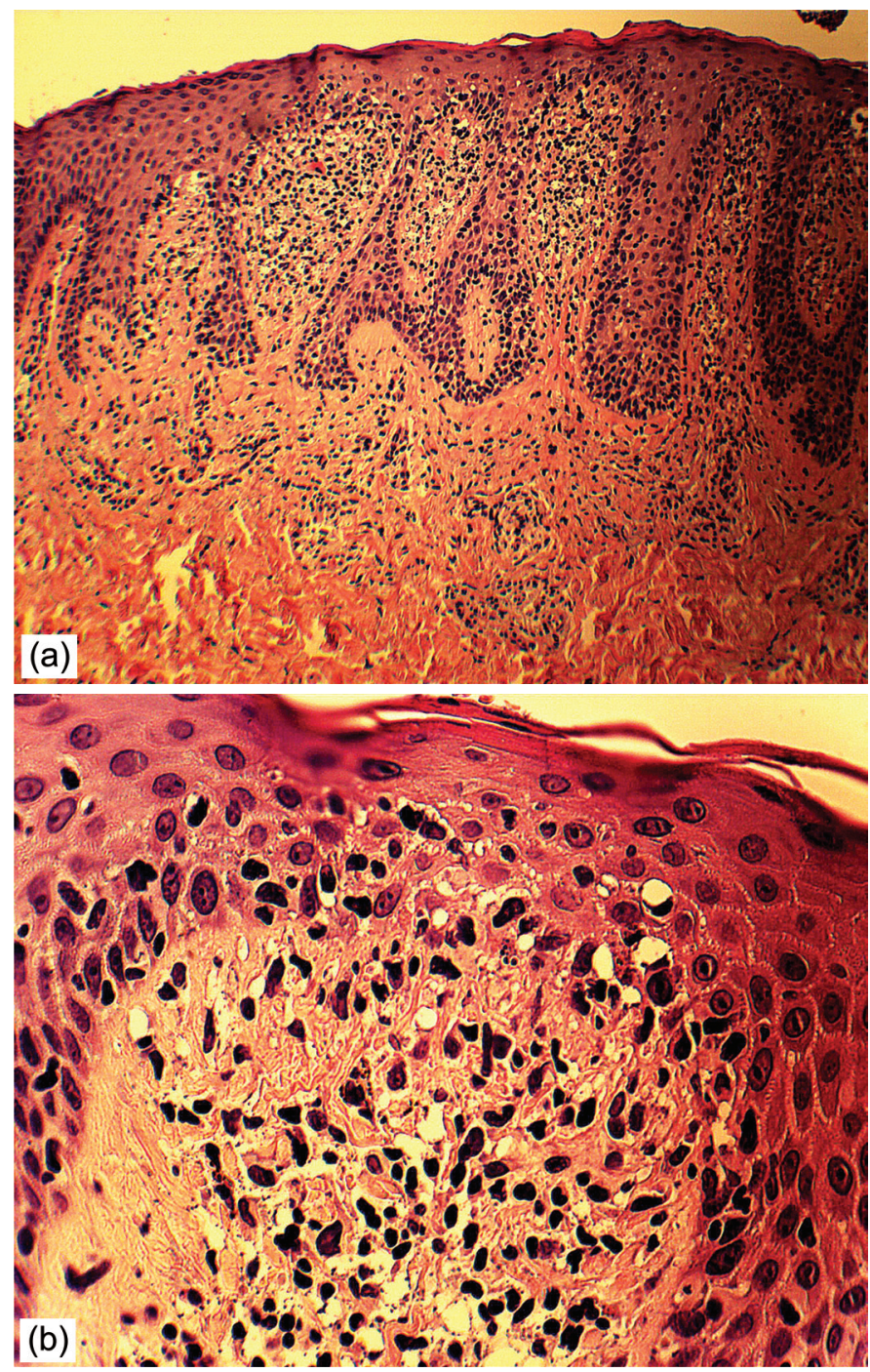

Figure 4 | First specimen taken from the erythematous patch on the anterior aspect of the chest (A: H\&E, $\times 160, B: H \& E, \times 640)$.

The second specimen with poikilodermatous changes revealed thinned, atrophic epidermis with mild hyperkeratosis and focal parakeratosis, focal spongiosis, and epidermotropism of atypical lymphocytes. There were an increased number of dilated blood vessels with lymphohistiocytic infiltrate surrounding them. An increased pigmentation of the basal layer of the epidermis and prominent melanin incontinence with melanophages were found (Fig. 5).

The third specimen was of particular interest. The findings
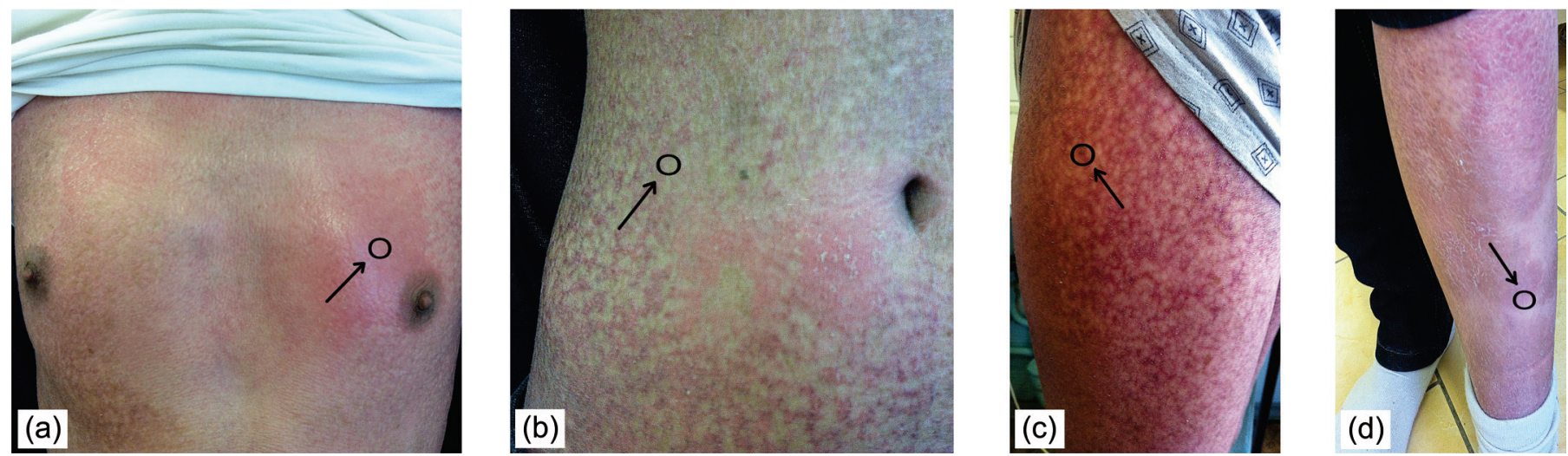

Figure 3 | Areas from which punch biopsy specimens were taken. (a) Erythematous patch on the anterior aspect of the chest; (b) Poikilodermatous patch on the right flank; (c) Lichenoid papule on the right thigh; (d) Plaque on the anterior aspect of the left shin. 

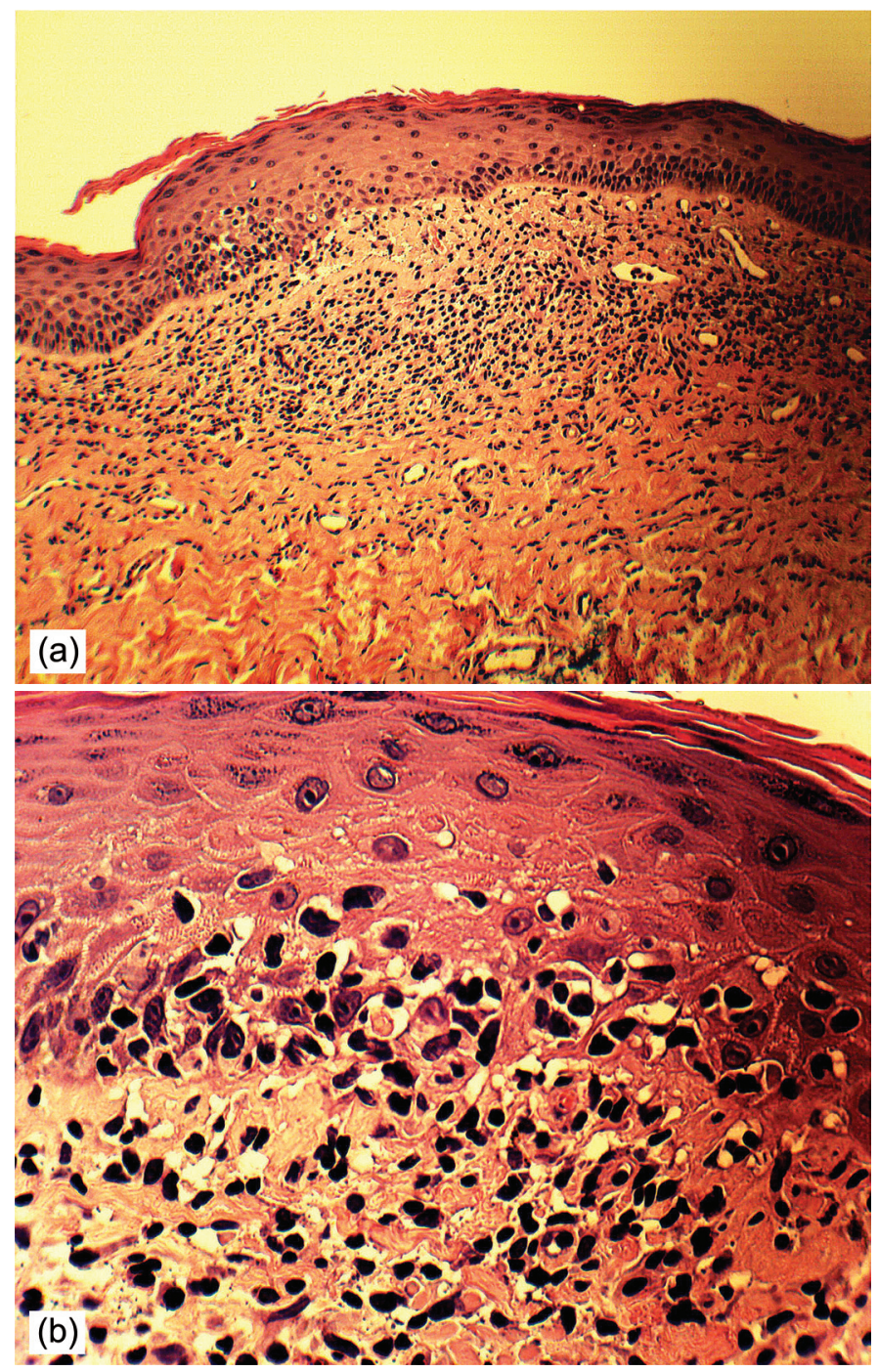

Figure 5 | Second specimen taken from the typical poikilodermatous patch on the right flank (A: H\&E, $\times 160, B: H \& E, \times 640$ ).

were very similar to those found in lichen ruber planus. They comprised such characteristic features as lymphohistiocytic bandlike infiltrate occupying the upper dermis and obscuring the dermoepidermal junction, irregular acanthosis resembling the typical saw-toothed appearance, extensive liquefactive degeneration of the basal layer of the epidermis with subepidermal clefts (Max Joseph spaces), pigmentary incontinence, and numerous cytoid bodies forming huge clusters. Nevertheless, in lichen planus the enumerated signs are usually seen along the entire specimen, whereas in ours they were distributed in a very well-organized, repetitive pattern showing the focuses with apparent histopathological changes alternating with relatively spared areas. In addition, a close-up view revealed atypia of the lymphocytes (Fig. 6).

Finally, the histological findings received from the biopsy on the plaques from the patient's shin corresponded to the classic patch or early plaque stage of MF. They included slightly acanthotic epidermis with occasional necrotic keratinocytes, basal cell hydropic degeneration, perivascular lymphohistiocytic infiltrate with the presence of atypical lymphoid cells, and overt epidermotropism (Fig. 7).

The immunohistological studies of all the specimens also revealed an unusual pattern with simultaneous presence of both CD4+ and CD8+.

Suspecting misdiagnosis, it was decided to reassess the biopsy findings that had been received in 2012 with additional sectioning of the preserved paraffin blocks. An appraisal of both slides was
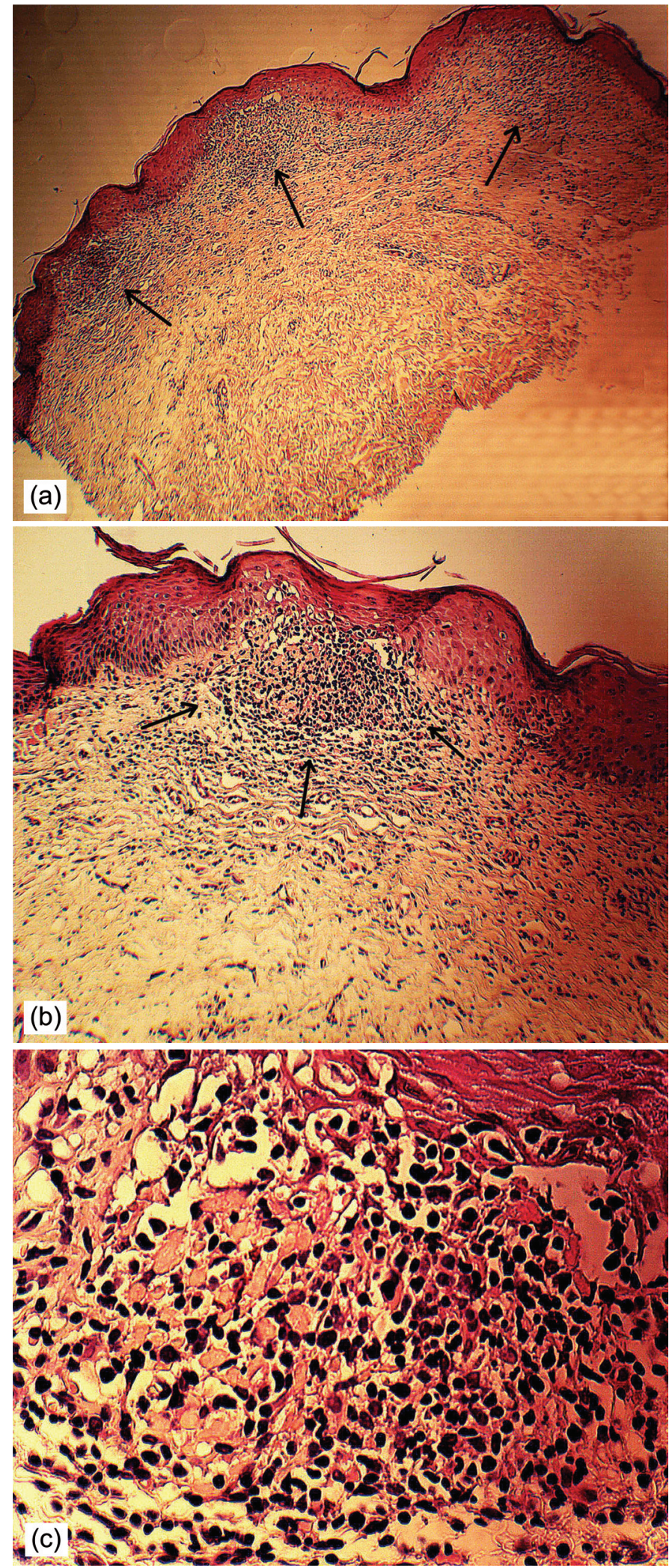

Figure 6 | Third specimen taken from the lichenoid papule on the right thigh. Note the unusual pattern of repetitive focuses of lymphohistiocytic infiltrate alternating with the relatively spared areas (A: $\mathrm{H} \& \mathrm{E}, \times 64, \mathrm{~B}: \mathrm{H} \& \mathrm{E}, \times 160, \mathrm{C}: \mathrm{H} \& \mathrm{E}$, $\times 640$ ).

made. Although the first one made 3 years ago showed the histopathological features of lichen planus, the second one received by additional sectioning revealed the signs of MF, although they were not apparent.

\section{Discussion}

Although the exact duration of MF in our patient could not be es- 


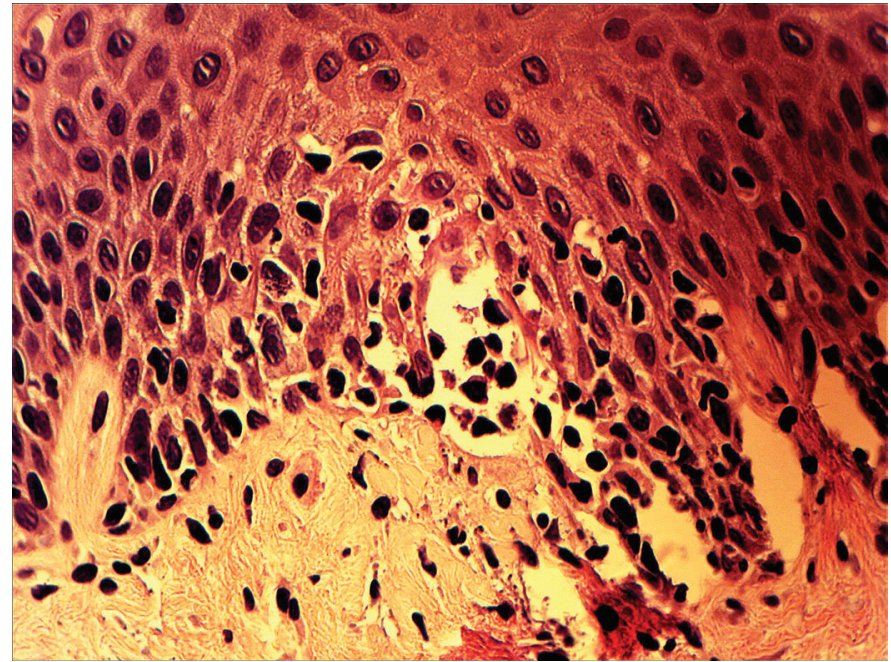

Figure 7 | Fourth specimen taken from the plaque on the anterior aspect of the left shin (H\&E, ×640).

tablished (because tests such as biopsy and immunohistochemistry were not performed at the time of the onset of his disease), we can state that 3 years ago he definitely received that diagnosis. The patient's medical history also highly suggests the presence of the disease from the very beginning of the case. His first complaints (asymptomatic hypopigmented patches or macules), which were regarded as vitiligo, in fact correspond to the diagnosis of hypopigmented variant of MF. Despite its extremely rare occurrence, in children and adolescents it can be seen more often. However, a natural reluctance to diagnose MF in children in addition to its clinical resemblance to vitiligo, tinea versicolor, pityriasis alba, or postinflammatory hypopigmentation often result in misdiagnosis.

The further development of the patient's symptoms and the replacement of hypopigmentation by hyperpigmentation can also be easily explained by the underlying histological changes. One of the main diagnostic pointers at any stage of MF is palisading of lymphocytes along the basal layer of the epidermis, usually accompanied by extensive basal cell hydropic degeneration. Research has shown that any damage to the basal layer of the epidermis and the entire area of the basal membrane (e.g., basal cell liquefactive degeneration) may cause pigment incontinence, which clinically manifests as hyperpigmentation. Consequently, the progression of pathological processes in the patient's skin resulted in a naturally determined appearance of pigmentation within the lesions.

When our patient developed papules within the pigmented patches, they were interpreted as lichen ruber planus based on the histological data. Unfortunately, it is not uncommon when lichen planus is histologically confused with MF. The bandlike infiltrate obscuring the dermoepidermal junction with cytoid body formation, confluent hyperkeratosis, irregular acanthosis, basal cell liquefactive degeneration, and apoptosis may closely mimic lichen planus. However, in our case at that stage misdiagnosis could have been avoided if additional block sectioning with subsequent immunohistochemical staining had been performed.

The case described here might be of particular interest for many dermatologists because it demonstrates very unusual clinical behavior of MF, in which one its rare variants is transformed into another that imitates benign dermatoses, thus posing definite obstacles in making a precise diagnosis.

To summarize, the diagnosis of MF and its rare atypical variants in particular is usually not obvious. Diagnostic mistakes might occur because of the clinical and in some cases histopathological similarity between MF and common benign dermatoses; for example, lichen ruber planus, morphea, which are dermatoses that can be associated with hypo- or hyperpigmentation, and other disorders.

Consequently, scrupulous assessment and juxtaposition of all the data collected, paying attention to the very minute details, may help to make the diagnosis more evident.

The histopathological features of the early stages of MF are often subtle or mimic other dermatoses; as a result, they can easily be overlooked. Thus, multiple biopsies with additional block sectioning and immunohistochemistry may be essential for obtaining an accurate diagnosis. Moreover, prior treatment before the biopsy masks characteristic features. Therefore, careful clinicopathological correlation is the only clue for the right diagnosis.

\section{References}

1. Calonje E, Brenn T, Lazar A, McKee PH. McKee's pathology of the skin with clinical correlations. 4th ed. Edinburgh: Elsevier Saunders; 2012: p. 1819.

2. Elder DE, Elenitsas R, Johnson BL Jr., Murphy GF, Xu X. Lever's histopathology of the skin. 10th ed. Philadelphia (PA): Lippincott Williams \& Wilkins; 2009: p. 1280 .

3. James WD, Berger TG, Elston DM. Andrews' diseases of the skin. Clinical dermatology. 10th ed. Edinburgh: Saunders Elsevier; 2006: p. 972.

4. Kreuter A, Hoffmamm K, Altmeyer A. A case of poikiloderma vasculare atrophicans, a rare variant of cutaneous T-cell lymphoma, responding to extracorporeal photopheresis. J Am Acad Dermatol. 2005;52:706-8.

5. Kikuchi A, Naka W, Harada T, Sakuraoka K, Harada R, Nishikawa T. Parapsoriasis en plaques: its potential for progression to malignant lymphoma. J Am Acad Dermatol. 1993;29:419.

6. Burg G, Dummer R, Nestle F.O, Doebbeling U, Haeffner A. Cutaneous lymphomas consist of a spectrum of nosologically different entities including mycosis fungoides and small plaque parapsoriasis. Arch Dermatol. 1996;132:567-572.
7. Lindae ML, Abel EA, Hoppe RT, Wood GS. Poikilodermatous mycosis fungoides and atrophic large plaque parapsoriasis exhibit similar abnormalities of $\mathrm{T}$ cell antigen expression. Arch Dermatol. 1988;124:366.

8. Abbott RA, Sahni D, Robson A, Agar N, Whittaker S, Scarisbrick JJ. Poikilodermatous mycosis fungoides: a study of its clinicopathological, immunophenotypic and prognostic features. J Am Acad Dermatol. 2011;65:313.

9. Farley-Loftus R, Mandal R, Latkowski JA. Poikilodermatous mycosis fungoides. Dermatol Online J. 2010;16:8.

10. Brecher A. Mycosis fungoides. Dermatol Online J. 2003;9:23.

11. Howard MS, Smoller BR. Mycosis fungoides: classic disease and variant presentations. Semin Cutan Med Surg. 2000;19:91.

12. Pimpinelli N, Olsen EA, Santucci M, Vonderheid E, Haeffner AC, Stevens S, et al. Defining early mycosis fungoides. J Am Acad Dermatol. 2005;53:1053-1063.

13. Dougherty J. Poikiloderma atrophicans vasculare. Arch Dermatol. 1971;103:550 